\title{
An Implicit Finite-Difference model for Thermal Processes Simulation and Surveys
}

\author{
by G. Ghodsi, D. Gavrilov*, E. Maeva* and R. Gr. Maev ${ }^{* *}$ \\ * Dept. of Physics, University of Windsor, Windsor (Ontario), Canada, N9B 3P4 \\ ** Institute for Diagnostic Imaging Research, Windsor (Ontario), Canada, N9A 5R5
}

\section{Abstract}

A finite-difference model based on the implicit approach for simulations of basic thermographic experiments is discussed. A specific sparse-matrices format was successfully utilized in order to accelerate the solution of a large system of equations while simulating each time step, as well as reducing the computer memory consumption.

By using the approach discussed, the authors demonstrate the applicability of the model for simulation of the Parker method for thermal diffusivity measurement in a 1D Cartesian, 2D Cartesian and cylindrical coordinate system. The model has demonstrated a good agreement with experiments conducted on samples of metals, epoxy and plastics.

\section{Introduction}

A numerical modeling of complex systems is a promising technique for prediction of behaviour of a sample under a real experiment. There are several techniques such as finite element and border element modeling. For samples with plane shape probably the simplest approach is finite difference model. In this work the finite difference approach was used in order to create a model for simulation of conduction of thermographic experiments. The model was applied to simulation of the Parker's method of thermal diffusivity measurement [1].

\section{Theory}

Finite difference approach is based upon an approximation of derivatives with ratios of finite changes of a function. In application to one-dimensional (1D) heat equation, this means that for a sample with uniform properties,

$$
\frac{\partial T(x, t)}{\partial t}=\alpha \frac{\partial^{2} T(x, t)}{\partial x^{2}} \Rightarrow \frac{T(x, t+\Delta t)}{\Delta t}=\alpha \frac{T(x+\Delta x, t)-2 T(x, t)+T(x+\Delta x, t)}{(\Delta x)^{2}}
$$

or

$$
\frac{\partial T(x, t)}{\partial t}=\alpha \frac{\partial^{2} T(x, t)}{\partial x^{2}} \Rightarrow \frac{T(x, t)}{\Delta t}=\alpha \frac{T(x+\Delta x, t+\Delta t)-2 T(x, t+\Delta t)+T(x+\Delta x, t+\Delta t)}{(\Delta x)^{2}}
$$

Depending on which equation - (1) or (2) is chosen for approximation, the approach is called explicit or implicit correspondingly. Although explicit approach is generally faster and simpler, it has a major drawback - it's conditional numerical stability. In order to overcome this constraint, implicit approach can be used.

Solving equation (2) for a large sample is equivalent to solving a matrix equation:

$$
A \cdot T^{\text {new }}=T^{\text {old }}
$$

where $T^{\text {old }}$ and $T^{\text {new }}$ correspond to temperature distributions over the sample at two adjacent time steps.

As it can be demonstrated, $A$ is a large square sparse matrix with three (in case of 1D situation), five (2D case) and seven (3D situation) diagonals. The size of $A$ increases rapidly with increasing of the simulated sample dimensions, so, even for a minor-size sample the number of elements in $A$ becomes too large for $A$ to be easily stored and processed in memory of computer.

In order to reduce the memory consumption and increase the speed of processing, one can use another format of matrix representation, where just non-zero elements of the matrix are kept as well as two auxiliary indices arrays, representing a portrait of the matrix [2]. In case of Cartesian coordinates the matrix $A$ is symmetric so the format can be simplified and designed to store only the upper triangle of the matrix. 
In case if the simulated experiment is considered axial-symmetric, one can simulate the only "slice" of the sample, which turns the $3 \mathrm{D}$ problem into $2 \mathrm{D}$. It can be shown though that in cylindrical coordinate system matrix $A$ is not symmetric anymore and the sparse matrix storage format should be adjusted to fit this situation.

The developed procedures for processing sparse matrices were applied to simulation of well-known Parker's method for thermal diffusivity measurement. This method assumes the study of temperature evolution of the back surface of the sample after the front surface is stimulated with a heat pulse [1].

\section{Setup}

In order to produce a short heat pulse the Speedotron 4803CX flash system was used providing a pulse energy up to $4800 \mathrm{~W} \cdot \mathrm{s}$. The flash was triggered via relay, which allowed for starting recording the temperature profile at the moment of the initiation of the flash.

For recording the temperature evolution profile the authors used FLIR SC4000 mid-infrared thermal imager with a spectral sensitivity in 3-5 $\mu \mathrm{m}$ band. The achievable framerate reached $400 \mathrm{fps}$.

\section{Results}

The simulation demonstrated that the temperature of the back side of the sample changes according to the predictions and its shape is suitable for the calculations of the thermal diffusivity (Fig.1.).
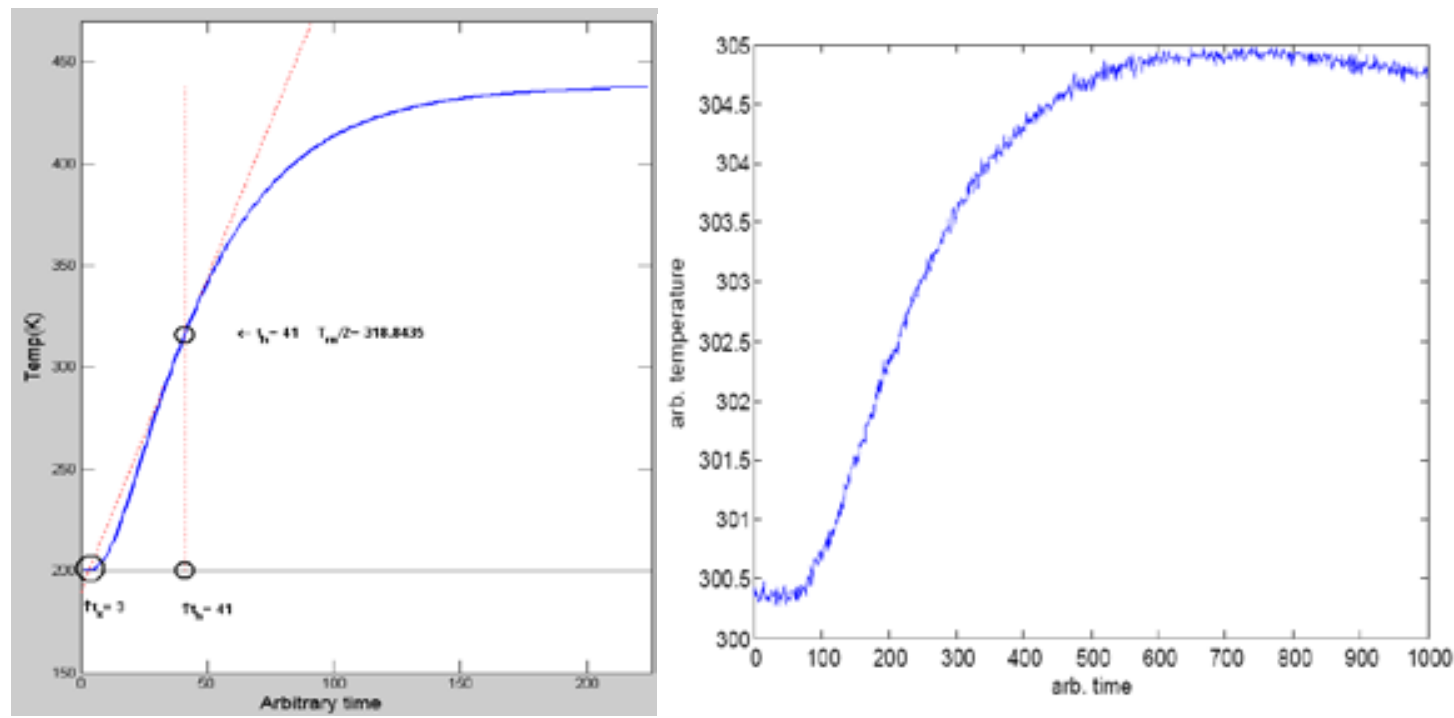

Fig. 1. Temperature evolution of the reverse side of a simulated sample (left)

and a curve obtained in experiment (right).

Other experiments conducted on layered samples and their simulations included studies of periodic heating conditions, pulse heating conditions, as well as simulations of the results of Pulse Phase thermography. The results of simulations and the experiment demonstrated good agreement, which confirms the viability of the model created.

\section{REFERENCES}

[1] Parker W. J., Jenkins R. J., Butler C. P. and Abbott G. L., "Flash Method of Determining Thermal Diffusivity, Heat Capacity and Thermal Conductivity", Journal of Applied Physics, 32(9), pp.1679-1684, 1961.

[2] Pissanetzky S., Sparse Matrix Technology. Academic Press, London, 321 p., 1984. 